\title{
THE PHYSIOLOGY OF PAIN
}

\author{
R. W. Gerard \\ Mental Health Research Institute, University of Michigan, Ann Arbor, Mich.
}

A decade ago I had occasion to review the problem of pain in an article that was not noticed by many neurophysiologists. In looking over more recent literature I was pleased to find that the kind of approach and picture that I then suggested remains essentially valid, despite considerable additions to our knowledge of the pain pathways, chiefly within the central nervous system. The material presented here is based on my earlier review, ${ }^{1}$ supplemented by a later, more general one, ${ }^{2}$ and by Sweet's chapter on pain in the Handbook of Physiology. ${ }^{3}$ Other recent reviews are the Ciba Foundation monograph ${ }^{4}$ and Barber's article in the Psychological Bulletin. ${ }^{5}$

The lack of real advance in new understanding, including learning how pain emerges into consciousness, is not due to any lack of importance of the subject or of interest in it. I remember well my experiences as an intern at Los Angeles General Hospital, Los Angeles, Calif., over one third of a century ago. We had many Mexican patients, and the only words of Spanish that I learned, I regret to say, were "Donde duele?,"* which sufficed. The essential point that I shall emphasize, one already alluded to by other contributors to this monograph, is the very real difference between awareness of pain and suffering from it. This distinction is of major importance clinically and pharmacologically, and we are now fairly close to understanding it neurophysiologically.

It is possible, on stimulating pain afferents by applying painful stimuli, to produce reflex responses such as changes in skin resistance at intensities below those required to produce any conscious awareness of them. Conversely, with stronger stimuli or other conditions, one can obtain a perfectly clear awareness of pain and yet not be particularly distressed by it. This condition can be achieved, for example, even with very severe pains, by procedures such as frontal leukotomies or topectomies. The sufferer with causalgic pain and allied ills may remain perfectly aware of feeling severe pain, but may no longer be really distressed by it; probably morphine acts in this manner. Although somewhat debatable, the best evidence I know of indicates that morphine does not raise the threshold of pain receptors to peripheral stimulation. The messages come into the nervous system as before, but they no longer seem as important as formerly.

There is, of course, a tremendous variability in the reaction of a given individual to a given painful stimulus, depending upon what can be called, loosely, the internal emotional state and the total external input (aside from the pain stimulus). This variation involves some of the mechanisms that I shall discuss. I suspect that the reader has had the experience of a toothache that seemed insufferable in the forlornness of late night, but became relatively minor the next morning, especially when facing the dentist's chair. Conversely, in the intense emotion of battle, only about one third of the soldiers who are severely wounded require analgesic medication whereas, in the same age group of males in civilian

* "Where does it hurt?" 
life suffering less severe trauma in surgical operations, 80 per cent do require such drugs. Let us examine the mechanisms involved.

Pain, of course, is of the utmost importance to the organism. In those rare individuals who lack perception of it, or when this perception is lost in disease, as in leprosy or tabes, repeated severe insults to the body occur, and major destruction results. Pain receptors are everywhere, although an occasional review or article still states that skeletal muscle, for example, or some of the viscera, cannot give pain. The stretching of almost any tissue or organ that resists stretching can produce pain. Stretching the viscera and blood vessels is notoriously painful; conversely, their constriction, which involves contraction of the smooth muscle in their walls, also can cause pain. The same thing is true of skeletal muscle. Anyone who questions this should recall a leg cramp, which is also related to contraction and stretching, or listen to a description of the pain of a muscle rupture. Pain afferents from skeletal muscle, as well as end tendons and joints, are known, and pain certainly can be elicited from joints and by stretching muscles under certain conditions; probably the Phillipson's and lengthening reflexes of the spinal animal are related to these pain receptors. The skin is sufficiently well known to require no further comment.

In all of these cases the sensitiveness to pain at the periphery is increased by the processes of inflammation. Whether these processes be mechanical or chemical or the pulsating effects of dilated vessels or some combination of all these is far from known, but certainly there is a marked exacerbation of pain receptivity from inflamed regions. This phenomenon deserves extensive study.

What neural mechanisms are involved? I know of no evidence that pain is perceived by any receptor other than a naked nerve ending. This does not mean that all naked nerve endings are pain receptors. It was long maintained, for example, that the cornea, which has only such naked endings, received only pain, but more recent evidence indicates decisively that temperature and a light touch can also be recognized from the cornea. The same thing is true for the eardrum and other regions; indeed, the fiber groups that carry pain (the $\delta$ and $C$ fibers) also mediate other sensations. However, the receptors for pain fibers are certainly the multiple undifferentiated naked endings scattered throughout the tissues and organs of the body. In the skin and cornea, where they have been studied carefully, it is now quite certain that these endings do not constitute an interlocking syncytial network of fibers, as was once believed. A given nerve fiber may serve a skin area of the order of $50 \mathrm{sq} . \mathrm{cm} .(5 \mathrm{~cm}$. by $10 \mathrm{~cm}$.), which is a large patch of skin, but many similar nerve fibers will also enter that same region so that their peripheries overlap and interlace; however, they do not form a continuum.

Considering next the afferent paths into the central nervous system: it is quite well established that 2 types of fibers carry pain, although not exclusively. The so-called $A \delta$, or $\delta$, elevation of the action spike, described in the original oscilloscope work that separated fiber types in a nerve trunk, is due to the relatively thinly medullated fibers, mostly 2 to $4 \mu$ in diameter, which conduct at a speed of 15 to $20 \mathrm{~m}$./ sec. They constitute about one third of the total number of medullated fibers in peripheral mixed nerves and certainly carry a type of pain. When the ending of 1 such fiber is given a single stimulus (a single electric shock over the skin ending is effective), one gets a subjective experience usually more 
like a tap than a prick, but when the same spot is tetanized a clear experience of pricking pain results. The other pain afferents are among the $\mathrm{C}$ fibers, which are very thin, nonmedullated fibers about $1 \mu$ in diameter and conducting at 1 to $2 \mathrm{~m}$. $/ \mathrm{sec}$., rather than $20 \mathrm{~m}$. $/ \mathrm{sec}$. There may be 4 times as many $\mathrm{C}$ fibers in a somatic nerve trunk as the total of medullated A fibers, and as many as 20 per cent of the fibers in a sympathetic trunk. The threshold for $\mathrm{C}$ fibers is as great as 100 times that of A fibers.

I stress these 2 kinds of fibers because there is no disagreement as to their existence and little as to their being related to the experience of pain. There is considerable disagreement in the literature, however, as to my next point. Many authors have described the double-pain experience after an appropriate stimulus, such as a quick, sharp pain and a slow, dull, aching or burning pain, and it has seemed reasonable to relate the rapid experience to conduction in the relatively fast $\delta$ fibers and the slow experience to conduction in the slow $\mathrm{C}$ fibers. Personally, I have no question about the 2 times and qualities, for I have never had difficulty in demonstrating the double pain on myself or to others (an easy method of doing this is to rest the shin on the seat of a chair with the unshod foot vertical, and give a smart rap to the sole with a flat piece of wood or a book). However, recent reviews, including that of Sweet, ${ }^{3}$ challenge the whole phenomenon as a figment of some experimenters' imaginations. Whatever the final answers may be, this dichotomy fits well into the over-all picture, and I shall use the double-pain system throughout this discussion.

Pain messages now enter the spinal cord. Those that come along $\delta$ fibers run up the ipsilateral dorsal column, primarily in relatively large fibers, and speed along at 50 to $80 \mathrm{~m}$. $/ \mathrm{sec}$. The messages that come in through $\mathrm{C}$ fibers travel in the crossed ventrolateral columns, in thin fibers, perhaps $2 \mu$ in diameter, and at a correspondingly low speed. The 2 systems of pain afferents thus continue to be quite distinctive. Indeed, the different systems have been traced into the midbrain, where they can be separately sectioned surgically; into the thalamus, with an average total conduction time (including synaptic delays) for the $\delta$ system of $8 \mathrm{~m}$./ $/ \mathrm{sec}$. and for the C system of $0.8 \mathrm{~m}$./ sec., again about one tenth; and, finally, to the somasthetic areas in the cortex, strongly to both I and II limb areas in cats, rabbits, and dogs, mainly to the I area in monkeys. On the way up, some fine fibers carry pain messages to the reticular formation, whence other fibers "alert" the cortex.

Now that pain messages have permeated the nervous system, I must be vague as to whether pain is experienced in the cortex or in subcortical structures. Fortunately, it may not matter a great deal, because there is such a strong reverberant circuit, a double feedback connection between the cortex, the thalamus, and other deep structures that activation entering that system might have the same outcome wherever it is related to conscious awareness. The intense generalized pain of thalamic lesions (the thalamic syndrome of Roussy and Dejerine), is to be recalled here, as well as the fact that cortical stimulation produces pain only rarely, and then chiefly when pathology is present.

Let us now return to my initial point concerning 2 basically different afferent systems. These are involved not only in pain, but also in experience in general. One system, relatively diffuse and related to the tone or set in which particularized patterned sensory experience is placed, includes the $C$ system; 
the other, giving the pattern, includes the $\delta$ system. Although the terms introduced by Head and Holmes have been violently attacked over the intervening decades, I find the words protopathic and epicritic extremely convenient. The protopathic is diffuse and vaguely discriminated, is strongly tinged by affect and, tending to summate and overflow when activated, is prepotent in determining the experience and the behavior of the individual. The epicritic experience, in contrast, is discrete, particulate, and localized and gives the patterned details of an experience, but with little affect. It has been happily compared with a picture on a television screen. The epicritic messages correspond to those that give the figures; the protopathic are like those (ordinarily unnoticed by the viewer when the set is well adjusted) that determine the setting and character-sweep speed, line pattern, level of contrast, total brightness, and other such characteristics.

The really important point is that these systems are, in one sense, antagonistic and, in another, complementary. For pain, explicitly, when the epicritic $\delta$ system is active, it may produce, to be sure, a clear awareness of pain (pricking, cutting, or other defined pain), but it does not lead to great suffering; rather, it inhibits the suffering induced by activation of the $\mathrm{C}$ protopathic system. Conversely, when the $\mathrm{C}$ system is active, it leads to all the suffering aspects of pain. I like to call it causalgic pain because it has many of the attributes so clearly recognized in causalgia, related to trigger points in general, and like aching. When this $\mathrm{C}$ system is active it facilitates the respones of cortical neurons to other pain impulses and whatever pain experience is involved. The epicritic system, conversely, and not only the pain but other modalities of input fibers such as those for touch and pressure, tend to suppress the protopathictype pain.

Next, let us consider a few experimental examples of these points. That pain is associated with the excitation of something is commonplace. Vast pains arise on the stimulation of trigger points. New trigger points can be generated by irritants applied to skin regions not ordinarily involved in the pain responses. Anginal attacks normally involve the left side; if the right chest has been irritated, as by cantharides concomitant with an anginal attack, future pain attacks may irradiate to the right as well as the left. The burning pain of putting a finger into ice water spreads to other fingers, not through peripheral connections, but centrally. Pervading pain is produced by stimulation through deep electrodes of the central gray matter or other parts of the old gray matter or of the afferent pathways to them. Causalgic pain. "leaks" past surgical lesions. Many other examples are cited in the earlier review. ${ }^{1}$ The evidence for pain on stimulation of the protopathic system is straightforward and need not be belabored.

Less familiar is evidence suggesting an inhibitory action of the epicritic system on this protopathic type of pain, but relevant data are plentiful. One of the earliest, and not unchallenged, findings was offered by $T$. Lewis and his colleagues. A pressure cuff on the arm produces a progressive block of peripheral nerve fibers; as conduction in the $\delta$ group disappears, the pain experienced suddenly develops a causalgic character. Lower-body ache or itch, such as pruritus ani, may be relieved by section of the the ventrolateral columns, but is severely exacerbated by section of the dorsal columns. Recently, surgical in- 
tervention in the midbrain has shown similar effects. Unilateral section of the epicritic-type paths gives a prompt hypalgesia, but in a few days there develops unilaterally a progressive burning ache that becomes agonizing and continuous.

In the periphery these systems can sometimes be manipulated to give, reversibly, pain or pain relief. The protopathic type of pain arises from the regenerating skin at an ulcer margin when only one pain fiber innervates it; when several fibers have entered, the aching type of pain disappears. A case has been reported in which, after a bullet wound in the arm, 3 different nerve trunks merged to innervate the same piece of skin on the hand; the subject had no pain or other difficulty. However, when 1 trunk was blocked by a local anesthetic, some tingling developed; when 2 were blocked, a severe causalgic pain appeared; and when all 3 were blocked, of course, anesthesia resulted. Anesthesiologists have met similar situations when using root-block anesthesia. One man, for example, had had severe amputation-stump pain 3 decades prior to an operation under spinal anesthesia. From the time the spinal roots became blocked until the anesthetic wore off he suffered agonizingly the ancient stump pain. Again, itch depends on $\mathrm{C}$ impulses and is relieved by rubbing, which activates $\delta$ fibers. A recent dramatic case report is comparable: a man with a continuous aching pain in the arm could always block it by squeezing the tips of his third and fourth fingers, so he kept his hand clenched to avoid pain; and this was not psychosomatic. Conditions such as causalgia and tic douloureux can often be overcome similarly by leading a patient to accept epicritic stimulation of the painful part.

The protopathic $\mathrm{C}$ system is the ancient, relatively undifferentiated one. It is constructed of short neurons with multiple synapses and wide connections and, probably, slow decremental conduction. These neurons possess relatively slow mobility, requiring much summation to activate them, but then give long after-discharges and long-enduring potentials. They interact through the fasciculi propriae in the central gray matter all along the primitive neuraxis; it is via such segmental paths that pain leaks past a chordotomy or similar surgical lesion of the principal pathways that were laid over the protopathic system later in evolution. Fortunately, these protopathic neurons are, on the whole, particularly sensitive to chemicals, both stimulating and depressing. The catechols, the indoles, most depressant agents, and other drug groups seem to act primarily on this system. In all these respects the epicritic elements are opposite.

With chronic states, that is the severe central causalgias, there develops what I have called a physiological inflammation. Continued and repeated protopathic inputs have built up excitation in central neurons by summation, irradiation, synchronous beating, reverberation in closed loops and, finally, fixation of a hyperactive state. As a guess, interneurons are mainly involved, but perhaps cortical pyramids, Golgi cells, and other structures participate. In any event, the elements related to pain experience become overactive and overactivable as a result of increased exciting bombardment, of decreased threshold, or of both. In both acute and chronic activation the C-fiber system acts on the pain-generating neurons to stimulate the protopathic structures directly or to sensitize them to patterned epicritic activation, presumably involving the cortex, as in the alerting responses from the reticular formation. Conversely, 
the $\delta$ system raises thresholds of the afferent channels so that protopathic impulses are blocked along the way.

A final few words on drug action, especially in relieving pain. A given agent may decrease the peripheral excitation and thus the afferent pain impulses. Where inflammation is involved, drugs such as the salicylates and cortisone may decrease the lesion and the pain. Where muscle spasm is the pain generator, especially if the skeletal system is involved, a relaxant can help; mephenesin and carisoprodol may contribute such an action. On the other hand, if the excitation is unchanged, the receptor threshold could be raised. This elevation is certainly, for several senses, under the influence of efferent nerve fibers, but I know of no evidence for it in the case of the simple pain receptors, nor of any drug that specifically depresses them. If pain messages reach the central nervous system, management of the condition is quite logical in principle: one may decrease protopathic flow by means of lesions or by raising thresholds of the appropriate neurons, or one may increase epicritic flow, which itself suppresses the protopathic transmission or neuron responses. In both cases the activation of the pain neurons is lessened.

At a meeting on carisoprodol held at Wayne State University, Detroit, Mich., last spring ${ }^{6}$ I was sufficiently foolish or sufficiently rational to suggest, simply on the basis of the clinical phenomena reported, that perhaps the action of this drug is to depress the reticular formation. I am pleased to see that work reported in this monograph makes that speculation seem less wild, perhaps, than it first seemed.

\section{References}

1. Gerard, R. W. 1951. The physiology of pain: abnormal neuron states in causalgia and related phenomena. Anaesthesiology. 12: 1-13.

2. Gerard, R. W. 1960. Molecules, neurons, and behavior. In Handbook of Physiology. Sect. 1. Neurophysiology. 3. J. Field, H. W. Magoun \& V. E. Hall, Eds. Am. Physiol. Soc. Washington, D.C. In press.

3. Sweet, W. H. 1959. Pain. In Handbook of Physiology. Sect. 1. Neurophysiology. 1: 459-506. J. Field, H. W. Magoun \& V. E. Hall, Eds. Am. Physiol. Soc. Washington, D.C.

4. Wolstenholme, G. E. W. \& M. O'Connor (Eds.). 1959. Pain and Itch. Nervous Mechanisms. Little, Brown \& Co. Boston, Mass.

5. Barber, T. X. 1959. Toward a theory of pain: relief of chronic pain by prefrontal leucotomy, placebos, opiates, and hypnosis. Psychological Bull. 56: 430-460.

6. Gerard, R. W. 1959. Summary of the Conference, in The Pharmacology and Clinical Usefulness of Carisoprodol. J. G. Miller, Ed. Wayne State University Press. Detroit, Mich.

\section{Discussion of the Paper}

Chauncey D. Leake (Ohio Stale University College of Medicine, Columbus, Ohio): To begin this discussion I shall ask Gerard to comment upon the efferent system in connection with pain.

Gerard. In so far as the spinal level is concerned, of course, my first reaction is to think back to the concept of pain quoted from Sherrington by Berger: "the physical adjunct of an imperative protective reflex." This is primarily the flexion reflex. The ordinary behavioral response to that kind of pain is a defensive or an avoidance movement. Such action, of course, makes use of the same motor system as every other act, so I doubt whether 
there is much point in attending to the efferent side in connection with pain, in contrast to any other motor response.

LEAKE. I have not made myself clear. How about the efferent sensory factor?

Gerard. Thank you; your question brings out a point about the feedback part of the mechanism that I went over too hastily. How does the epicritic type of impulse enter the situation and tend to inhibit the other type? There are 2 possible ways for the epicritic impulse, which is faster, to block the protopathic one and I rather suspect that both methods are involved. The epicritic impulses enter the nervous system more rapidly than do the protopathic ones and thus can modify the synaptic junction to make it less transmissive for the protopathic messages. The other way is by a straight feedback mechanism, the epicritic impulses going on up to higher centers and feeding back down on one of the way stations in the afferent path and raising pain thresholds there.

In general, one can control pain, either peripherally or centrally, only by decreasing the activity of those neurons whose activity is associated with pain. There are 2 ways of doing this: first, by decreasing the inflow of messages to excite those neurons and, second, by raising the pain thresholds of those neurons so that it takes more messages than before to excite them. All drugs must act in one or the other of these ways.

QuEsTion: Gerard mentioned certain terminology that, according to Lewis, would belong to deep pain. I wondered whether Gerard agrees with Lewis that there are 2 types of pain, one being skin or superficial pain and the other deep pain.

According to Lewis, skin pain is always sharp and pricking or bright, and it becomes burning only when it is longer in duration, whereas the definition of dull pain attributes it to the deep structures under the skin and thus differentiates it from skin pain.

Gerard. You are asking essentially for an anatomical separation, wherea; I gave a physiological and functional one. I question if burning, causalgic pain is limited to the deep structures; such pain can also be obtained from regenerating or ulcerated skin, or under other conditions in which the epicritic fibers from the skin are thrown partially out of function. 